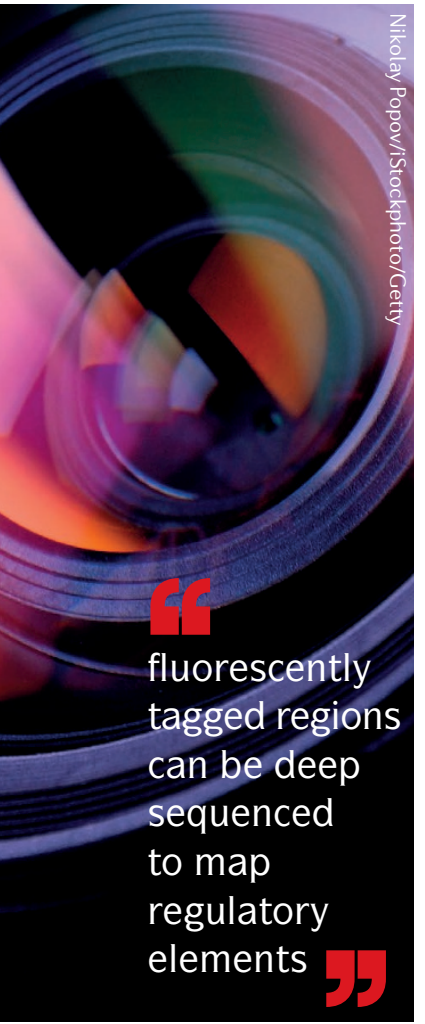

$\Rightarrow$ TECHNIQUE

\section{See and seq the regulome}

A new technique, named assay of transposase-accessible chromatin with visualization (ATAC-see), allows imaging and sequencing of the accessible genome in the same cells.

Existing epigenome analysis methods - such as DNase sequencing (DNase-seq), FAIRE-seq or ATAC-seq - probe open chromatin regions, which harbour active regulatory elements such as enhancers, promoters or insulators, outside of their nuclear context. In brief, the chromatin profiling method ATACseq is based on transposition of sequencing adapters into chromatin of living cells by hyperactive Tn 5 transposase. Sites tagged by adapters are isolated and sequenced to yield information on regulatory elements, transcription factor binding and nucleosome occupancy.
The newly developed technique ATAC-see modifies the existing ATAC-seq protocol into a transposase-mediated imaging technology through fluorescent labelling of accessible loci. This approach "transforms the molecular accessibility of chromatin into points of light," say the authors. Once the three-dimensional spatial organization of the open-chromatin landscape has been imaged, the fluorescently tagged regions can be deep sequenced to map regulatory elements as per the standard protocol.

Application of ATAC-see to five human cell types showed that the organization of euchromatin into higher-order structures is highly cell type-specific. Chen et al. also showcase the usefulness of a joined-up approach that combines molecular imaging and epigenomic sequencing using ATAC-see and ATAC-seq in the same cells, in human neutrophils. Using this approach, the team were able to visualize and decipher mechanistic steps of a form of programmed cell death used by neutrophils to kill bacteria through the release of chromatin, which is known as neutrophil extracellular traps or NETosis.

Finally, the authors integrated ATAC-see with flow cytometry, enabling quantitative cell counting and prospective cell sorting as a function of chromatin accessibility. Further studies using ATAC-see combined with fluorescence-activated cell sorting should yield insights into the contribution of chromatin states to cellular heterogeneity.

Linda Koch

ORIGINAL ARTICLE Chen, $X$. et al. ATAC-see reveals the accessible genome by transposasemediated imaging and sequencing. Nat. Methods http://dx.doi.org/10.1038/nmeth.4031 (2016) 